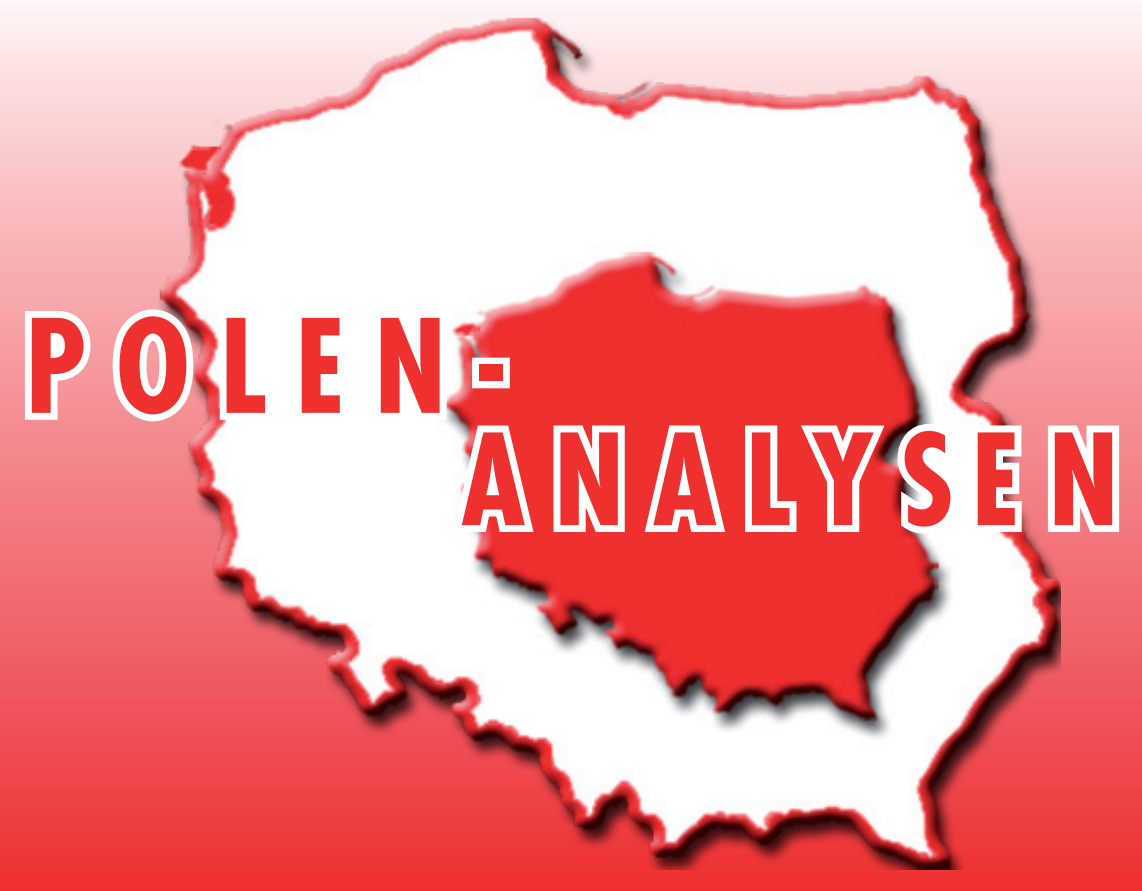

www.laender-analysen.de/polen

\title{
FRAUEN IN DER POLITIK
}

\section{ANALYSE}

Die Selbstverwaltungswahlen 2010 -

der Frauenanteil auf Listen und in Wahlen

Małgorzata Druciarek und Aleksandra Niżyńska, Warschau

- TABELLEN UND GRAFIKEN ZUM TEXT

Politische Partizipation von Frauen in Polen

\section{CHRONIK}

Vom 20. September bis zum 03. Oktober 2011 


\title{
Die Selbstverwaltungswahlen 2010 - der Frauenanteil auf Listen und in Wahlen
}

\author{
Małgorzata Druciarek und Aleksandra Niżyńska, Warschau
}

\section{Zusammenfassung}

Das im Januar 2011 verabschiedete Wahlgesetz sieht vor, dass sich auf einer Wahlliste nicht weniger als $35 \%$ Frauen und nicht weniger als 35 \% Männer befinden müssen, damit diese zugelassen werden kann. Schon im Wahlkampf zu den Regional- und Kommunalwahlen 2010 war das Thema der Teilhabe von Frauen an der Politik zu einer Schlüsselfrage geworden. In der Tendenz stellt die Analyse einen systematischen Anstieg des Frauenanteils unter den Kandidaten für politische Ämter fest, doch ging dieser Anstieg von Wahl zu Wahl zurück. Im Vergleich zu 2006 gab es bei den Selbstverwaltungswahlen 2010 nur um 2 Prozentpunkte mehr Kandidatinnen. Die 35\%-Regelung kann sich nach Auffassung der Autorinnen nur als effektiv erweisen, wenn die Parteien sich dazu entscheiden, Frauen auf den vorderen Plätzen der Wahllisten zu platzieren. Erste Ergebnisse der Quotenregelung wird man bereits nach den Parlamentswahlen am 9. Oktober 2011 sehen können - den ersten Wahlen, bei denen die neue Regelung gilt.

\section{Frauen in politischen Ämtern}

In den meisten Ländern Westeuropas erzielen Frauen bei Wahlen größere Erfolge auf lokaler Ebene als auf der des Gesamtstaates. Im Durchschnitt der 27 EUStaaten beträgt der Frauenanteil in den lokalen Legislativorganen $31 \%$, in den Nationalparlamenten hingegen nur $24 \%$. Diese Proportionen bleiben auch in der Exekutive erhalten: Auf der lokalen Ebene gibt es 32\% Politikerinnen, aber nur jedes vierte Regierungsmitglied ist eine Frau. Ausnahmen bilden dabei nur die skandinavischen Länder, die in den Rankings zur Partizipation der Frauen am öffentlichen Leben die vordersten Plätze belegen (u. a. Gender Gap Index 2010). In Finnland, Island und Schweden bewegt sich die politische Partizipation von Frauen in der überregionalen Politik um $50 \%$, in Dänemark und Norwegen erreicht sie fast $40 \%$. Viel ungünstiger ist dagegen die Situation für Kommunalpolitikerinnen. Bei Kommunalwahlen werden skandinavische Politikerinnen viel seltener gewählt als bei Parlamentswahlen. Ihr Anteil in der Kommunalpolitik liegt bei $20-30 \%$.

Polen unterschied sich diesbezüglich viele Jahre lang nicht vom europäischen Durchschnitt - der Frauenanteil in der Lokalpolitik war grundsätzlich höher als im Nationalparlament (Sejm). Doch vergrößerte sich, entgegen den in anderen Ländern zu beobachtenden Tendenzen, der Anteil von Politikerinnen in den Parlamenten der territorialen Selbstverwaltung ${ }^{1}$ nicht systematisch

1 Die territoriale Selbstverwaltung umfasst in Polen die Ebenen der Gemeinden, Kreise und Woiwodschaften. Die Grundeinheit bildet die Gemeinde mit dem Gemeinde- oder Stadtrat als Parlament. Die Exekutive bilden die Gemeindevorsteher (wójt), der Bürgermeister (burmistrz) oder der Stadtpräsident (prezydent miasta). Der Kreis (powiat) fasst mehrere Gemeinden zusammen: Der Kreisrat (rada powiatu) fasst Beschlüsse, der Kreisvorstand (starosta) setzt sie um. Auf der Ebene der Woi- von Wahl zu Wahl. Im Jahr 2002 kehrte sich, wenn auch nicht für lange, die gesamteuropäische Tendenz um. Denn nach den Wahlen zu den Parlamenten von den Woiwodschaften bis zu den Gemeinden zeigte sich, dass Frauen im Sejm zahlreicher vertreten waren als dort. Dies war so, obwohl in den Monaten vor den Wahlen alles darauf hingewiesen hatte, dass der Anteil der Politikerinnen steigen würde. Vor den Parlamentswahlen 2001 hatten die drei damals wichtigsten politischen Parteien - das Bündnis der demokratischen Linken (SLD), die Arbeitsunion (UP) und die Freiheitsunion (UW) den Grundsatzbeschluss gefasst, dass keines der beiden Geschlechter auf den Wahllisten mit weniger als $30 \%$ Kandidatinnen oder Kandidaten vertreten sein durfte. Die von den politischen Parteien eingeführten Quoten beeinflussten das Wahlergebnis, der Anteil der Frauen im Sejm wuchs signifikant. Es waren also Voraussetzungen dafür vorhanden, dass der Anteil der bei den Selbstverwaltungswahlen gewählten Frauen ebenfalls signifikant ansteigen würde. Doch dazu kam es nicht. Trotz der von manchen Parteien gefassten Beschlüsse über eine Mindestvertretung jedes Geschlechts auf den Wahllisten bestand eine beträchtliche Mehrheit der Wahlkandidaten zu den Selbstverwaltungsorganen aus Männern. Im Durchschnitt besetzten Frauen jeden vierten Platz auf den Listen für die Gemeinde- und Kreisräte. Wesentlich schlechter sah es aus bei den Kandidatinnen für die

wodschaft übt das Regionalparlament (sejmik województwa, in diesem Text teilweise auch als Woiwodschaftsparlament bezeichnet) die gesetzgebende Gewalt aus, während der Marschall (marszałek województwa) die laufenden Geschäfte tätigt. Von besonderer Bedeutung auf dieser Ebene ist eine "Doppelherrschaft« der Selbstverwaltung und der Zentralregierung, denn neben dem demokratisch legitimierten Marschall »regiert« dort auch noch ein von Warschau eingesetzter Regierungsbeamter, der so genannte "Woiwode». 
direkten Wahlen auf die Posten von Gemeindevorstehern, Bürgermeistern und Stadtpräsidenten: Hier stellten Frauen kaum mehr als $10 \%$ aller Kandidierenden.

Nach 2002 wuchs der Frauenanteil in der lokalen und regionalen Politik allmählich von Wahl zu Wahl. Aus den Daten der Staatlichen Wahlkommission geht hervor, dass $200218 \%$ der Mitglieder der Regional- und Kommunalparlamente Frauen waren, vier Jahre später war es schon jeder fünfte. Dieser Anteil stieg auf 25\% bei den Wahlen 2010, den letzten Wahlen vor Einführung der Quotenregelung im polnischen Wahlrecht.

Die Regional- und Kommunalwahlen 2010 hatten einen besonderen Charakter angesichts der in dieser Zeit intensiv geführten öffentlichen Debatte über die Paritäten, die im Sinne der Verteidiger dieser Forderung eine Steigerung des Frauenanteils in den politischen Ämtern der Selbstverwaltungsorgane bewirken sollten. Den Anstoß zu einer lebhaften Diskussion gab der bürgerschaftliche Entwurf eines Paritätsgesetzes im Dezember 2009. Die »Einführung eines Paritätssystems, das auf den Listen für die Wahlen aller Ebenen gilt, und eine Sanktion, die die Zulassung einer Liste verbietet, bei der dieser Grundsatz nicht eingehalten wird", war eine der Hauptforderungen des Ersten Frauenkongresses. Diese Forderung löste eine erbitterte Diskussion zwischen Befürwortern und Gegnern aus. Die Geschicke dieses bürgerschaftlichen Gesetzentwurfes wurden in den Medien das ganze Jahr 2010 über mit wechselnder Intensität kommentiert.

Allerdings war dies nicht der erste Versuch, eine Quote bei den Wahlen zu den Parlamenten und Entscheidungsgremien in Polen einzuführen. Das erste Mal versuchte man die Quote in dem Gesetz über den gleichen Status von Frauen und Männern festzuschreiben. Dann machte 2001 die Abgeordnete Olga Krzyżanowska den Vorschlag, die besagte Regelung in die Wahlordnung einzufügen. Beide Versuche misslangen. Etwas erfolgreicher verlief die bereits erwähnte Einführung von Quoten durch die drei wichtigsten politischen Parteien unmittelbar vor den Sejmwahlen von 2001. Jedoch erst das im Januar 2011 verabschiedete Wahlgesetz sieht vor, dass sich auf einer Wahlliste nicht weniger als $35 \%$ Frauen und nicht weniger als 35\% Männer befinden müssen, damit diese zugelassen werden kann. Sicher können wir nicht von einem Sieg der Urheberinnen der Forderung des Ersten Frauenkongresses sprechen - von dem bürgerschaftlichen Entwurf des Paritätsgesetzes ist nur die Verabschiedung eines Quotengesetzes übrig geblieben. Doch ganz gewiss hat die Einführung der 35\%-Quote eine lebhafte Diskussion über die Teilhabe von Frauen an der Politik ausgelöst. Das ist insofern wichtig für die Analyse des Frauenanteils in der Lokalpolitik, als in der öffentlichen Debatte, die den Selbstverwaltungswahlen von 2010 vorausging, Quoten und Paritäten den Ton angaben. Das Thema der Teilhabe von Frauen an der Politik war zu einer Schlüsselfrage in diesem Wahlkampf geworden. Hat sich das in spürbarer Weise auf den Wahlerfolg von Frauen ausgewirkt?

\section{Kandidatinnen bei den Regional- und Kommunalwahlen 2010}

Die Bewertung des Frauenanteils bei den Wahlen 2010 muss vor allem ansetzen bei der Analyse der Anzahl der Kandidatinnen, die von den einzelnen Wahlkommissionen aufgestellt wurden. Mit der steigenden Zahl von Kandidatinnen in den Wahlen auf allen Selbstverwaltungsebenen steigt auch die Anzahl gewählter weiblicher Ratsmitglieder. 2002 war unter den Kandidaten auf allen Selbstverwaltungsebenen kaum mehr als ein Viertel Frauen, 2006 stieg dieser Anteil auf 29\%, und 2010 überschritt er die 30\%-Marke.

In der Tendenz gibt es also einen systematischen Anstieg des Frauenanteils unter den Kandidaten für politische Ämter, doch geht dieser Anstieg von Wahl zu Wahl zurück. Im Vergleich zu 2006 gab es bei den letzten Wahlen 2010 nur um 2 Prozentpunkte mehr Kandidatinnen. Das kann bedeuten, dass die bisher verfolgten Strategien zur Steigerung des Frauenanteils bei den Wahlen unwirksam geworden sind und man entschiedenere Maßnahmen ergreifen muss.

In diesem Zusammenhang ist an die Typologie von Strategien zur Erhöhung des Anteils von Kandidatinnen bei Wahlen von Joni Lovenduski zu erinnern. Die britische Forscherin verweist auf drei grundsätzliche Möglichkeiten, die zu einer breiteren Nutzung des passiven Wahlrechts durch Frauen führen sollen:

- Rhetorik der Gleichheit - öffentliche Debatte um die Teilhabe von Frauen an der Politik, öffentliche Akzeptanz für eine Steigerung der Anzahl von Politikerinnen, ausgedrückt durch bekannte Persönlichkeiten, offene Ermutigung von Frauen zur Kandidatur;

- Förderung der Gleichheit - Organisation von Schulungen für Kandidatinnen, inhaltliche und finanzielle Hilfe im Wahlkampf;

- Garantien der Gleichheit - Regelungen, die die Anzahl der Frauen auf einer Wahlliste festschreiben, meist in Form von Quoten, die von Parteien intern festgelegt bzw. im Parlament verabschiedet werden, als bindendes Recht für alle Wahlkomitees, die Wahllisten aufstellen.

Bei den Wahlen 2010 wurde die Rhetorik der Gleichheit im Hinblick auf die oben beschriebene Diskussion über Paritäten und später über die Quotenregelung ausgiebig verwendet. Dazu wurde Werbung für die Gleich- 
heit betrieben, um die Anzahl der Wahlkandidatinnen zu erhöhen. Dank zahlreicher regionaler und überregionaler Schulungen konnten sich die Frauen Grundwissen über die territoriale Selbstverwaltung sowie über Techniken der Wahlkampfführung aneignen. Workshops über Selbstdarstellung, öffentliches Auftreten und erfolgreiche Kommunikation sollten den Frauen helfen, bestmögliche Wahlergebnisse zu holen, und die Kandidatinnen unterstützen.

Trotz dieser Aktivitäten wuchs der Anteil der Kandidatinnen im Vergleich zu den vorangegangenen Wahlen nur um 2 Prozentpunkte. Offenbar ist es auf dieser Ebene der Partizipation von Kandidatinnen wichtig, eine Strategie anzuwenden, die Lovenduski als »Garantie der Gleichheit» bezeichnet, also Quotenregelungen einzuführen, die eine bestimmte Anzahl von Plätzen für Frauen auf der Wahlliste des jeweiligen Komitees sicherstellen. Die im Wahlgesetz von 2011 verabschiedete Quote von mindestens $35 \%$ für beide Geschlechter wurde 2010 von keiner der im Parlament vertretenen Parteien auf Frauen angewendet.

Keine Partei erreichte auch nur einen Frauenanteil von $30 \%$ auf ihren Listen. Diesem Wert näherten sich lediglich SLD und Bürgerplattform (PO) mit jeweils $29 \%$ Frauenplätzen auf ihren Listen an. Am wenigsten Frauen fanden sich auf der Liste der Partei Recht und Gerechtigkeit (PiS): Für Kandidatinnen dieser Partei gab es dort gut ein Fünftel der Plätze. Doch ist nicht nur die Präsenz von Frauen auf der Liste von Bedeutung, sondern es sind auch die Plätze, von denen aus die Kandidatinnen starten. Gerade von diesem Faktor hängt in hohem Maße ihr Wahlerfolg ab. Vergleicht man die Anzahl der Frauen, die für verschiedene Parteien in die Regionalparlamente der Woiwodschaftsebene gelangten, kann man jedoch zu der Schlussfolgerung kommen, dass die Bedeutung eines Listenplatzes bei einigen Parteien wichtiger ist als bei anderen.

Bei der Untersuchung der Listen der einzelnen Parteien ist festzustellen, dass die Listen der PO den höchsten Anteil von Frauen auf den Plätzen 1-5 aufwiesen ( $43 \%$ ), danach folgte mit $41 \%$ gleich die Bauernpartei (PSL). Am wenigsten Kandidatinnen auf den vorderen Plätzen hatten Recht und Gerechtigkeit (35\%), etwas mehr waren auf den Listen der SLD zu finden (38\%). Betrachtet man jedoch den Frauenanteil unter den auf den Listen der jeweiligen Partei gewählten Ratsmitgliedern, stellt man fest, dass Frauen am erfolgreichsten von den Listen der SLD gestartet sind, obwohl diese einen geringeren Frauenanteil auf den vorderen fünf Listenplätzen aufweist als PO und PSL. Trotzdem stellen Frauen in zwei Woiwodschaften die Hälfte der SLD-Ratsmitglieder dar. Wie ist es zu erklären, dass ausgerechnet auf den Listen dieser linken Partei am meisten Frauen in die Regionalparlamente gelangten?

Ein Schlüsselfaktor dafür war wohl die Verbindung zwischen einem auf offenen Listen basierenden Wahlsystem und der positiven Einstellung der Wählerschaft der SLD zur Präsenz von Frauen in der Politik. Dank der offenen Listen können die Wähler selbst über die endgültige Reihenfolge der Personen entscheiden, die ins Regionalparlament kommen, und nicht die Parteifunktionäre, die die Listen aufstellen. Ins Regionalparlament kann also jemand kommen, der auf Platz 14 der Liste steht, während jemand auf Platz 2 scheitern kann, wenn er weniger Stimmen erhält. In der Praxis werden meist die Stimmen für die Personen auf den vorderen Plätzen der Liste abgegeben, denn wenn die Wähler in die Wahllokale kommen, wissen sie meist noch nicht, wen sie konkret wählen werden. Wird jedoch die Wählerschaft einer Partei für die Wahl von Frauen mobilisiert, besteht die Chance, deren Anteil in den Regionalparlamenten unabhängig von ihrem Listenplatz zu erhöhen. So war es höchstwahrscheinlich bei der SLD.

Wenn die Wähler einer Partei wiederum der Präsenz von Frauen in der Politik nicht wohlgesonnen sind, sind geschlossene Listen für die Kandidatinnen vorteilhafter, auf denen ihr Platz von vornherein feststeht und von denen aus sie aufgrund einer entsprechenden Anzahl der für diese Liste von abgegebenen Stimmen in die Entscheidungsgremien gelangen können. Natürlich nur, wenn die Parteigremien sich entscheiden, Frauen auf den vorderen Plätzen aufzustellen, was nicht oft geschieht. Bei einer allgemeinen gesellschaftlichen Ablehnung der Teilhabe von Frauen in der Politik kann daher die Einführung von Quoten auf geschlossenen Listen eine effektive Möglichkeit sein, ihnen diese zu verschaffen, unter Anwendung des so genannten Reißverschlussprinzips, d. h. der abwechselnden Platzierung von Frauen und Männern auf der Wahlliste.

Die größte Bedeutung hat die Politik der Parteien bezüglich des Frauenanteils in den Gremien bei den Wahlen zum Woiwodschaftsparlament. Für die Vertretungen von Gemeinden unter 20.000 Einwohnern kandidieren auf den Parteilisten nur $31 \%$ Frauen, für die Woiwodschaftsparlamente werden gar drei Viertel der Frauen von den Parteien aufgestellt. Angesichts der Art der Machtausübung auf dieser Ebene verwundert das nicht. Dort agieren sowohl die demokratisch legitimierte Selbstverwaltungsebene als auch die Regierungsebene in Gestalt des vom Ministerpräsidenten nominierten Woiwoden. Die Selbstverwaltung der Woiwodschaft entscheidet über Schlüsselfragen für die Entwicklung der Region: die Verteilung von EU-Mitteln, den Ausbau von Verkehrswegen, den Umweltschutz. Die enge Zusammenarbeit mit der Regierungsverwaltung auf 
dem Gebiet der Woiwodschaft hat zur Folge, dass die Politiker auf dieser Ebene eher gesamtstaatlichen »Playern« als lokalen »Aktivisten« ähneln. Daher nährt die Tatsache der systematischen Vergrößerung der Frauenrepräsentanz in den Regionalparlamenten die Hoffnung auf einen Anstieg des Frauenanteils auch in der Politik des Gesamtstaates.

Innerhalb von 12 Jahren ist der Frauenanteil in den Woiwodschaftsparlamenten um 12 Prozentpunkte gestiegen. 2010 wurden $23 \%$ der Mandate in diesen Parlamenten von Frauen besetzt. Festzustellen ist, dass wenn der Anteil der Kandidatinnen auf den Wahllisten in einer bestimmten Region sich dem 30\%-Wert nähert oder diesen erreicht hat, sich der Frauenanteil im Regionalparlament auf demselben Niveau hält. Dies zeigt, dass die 35\%-Quote die Chance bietet, die Repräsentanz der Frauen auf dem Niveau der so genannten kritischen Masse, d. h. bei $30 \%$, zu sichern. Untersuchungen belegen, dass erst ein solcher Anteil von Vertretern einer Gruppe in Entscheidungsgremien gewährleistet, dass die Perspektiven der Mitglieder dieser Gruppe auch berücksichtigt werden.

Bei der zusammenfassenden Betrachtung des Frauenanteils in den Entscheidungsgremien nach den Regional- und Kommunalwahlen von 2010 bemerken wir eine deutlich höhere Partizipation von Frauen auf der untersten Ebene, also in den Gemeinden bis 20.000 Einwohner, in denen das Mehrheitswahlrecht galt. Unter den Ratsmitgliedern auf dieser Ebene waren $26 \%$ Frauen. Ein etwas geringerer Anteil von Politikerinnen sitzt in den Räten der kreisfreien Städte (24\%). Einen vergleichbaren Frauenanteil weisen auch die Woiwodschaftsparlamente und Gemeinderäte auf. Am wenigsten Frauen wurden in die Räte der Kreise gewählt: Nur $18 \%$ aller gewählten Mitglieder dieser Ebene waren weiblichen Geschlechts.

Ausgehend von diesen Daten ist schwer zu prognostizieren, ob die Tendenz einer systematischen Zunahme der Repräsentanz von Frauen in den lokalen und regionalen Entscheidungsgremien weiter andauern und sich bei den Wahlen 2014 bestätigen wird. Die Verabschiedung des Wahlrechts wird sich zweifellos auf die Proportionen der Geschlechter in den Entscheidungsgremien auf allen Ebenen der Selbstverwaltung auswirken, doch die Richtung dieser Veränderung ist vorerst schwer einzuschätzen. Erstens wird die 35\%-Quotenregelung für Frauen und Männer gelten, deren Ziel die Steigerung des Frauenanteils in der Politik ist. Ob sich diese Regelung ohne eine Garantie für bestimmte Frauenplätze auf der Liste als effektiv erweist, wird man bereits nach den Parlamentswahlen am 9. Oktober 2011 sehen können - den ersten Wahlen, bei denen die neue Regelung gilt.
Zweitens umfasst nach dem neuen Gesetz das Verhältniswahlrecht, in dem Parteilisten verpflichtend sind, nur die beiden höchsten Ebenen der Selbstverwaltung: den Kreis und die Woiwodschaft. Wahlen zu den Gemeinderäten, die nach dem Wahlgesetz unabhängig von ihrer Größe auf dem Mehrheitswahlrecht mit Einmandatswahlkreisen basieren, bleiben ohne Quotenregelungen. Denn die Parteien stellen nur einen Kandidaten oder eine Kandidatin auf. Diese Lösung ist weder für Frauen noch für andere in der Politik unterrepräsentierte Gruppierungen günstig. Außerdem hat Präsident Bronisław Komorowski angekündigt, er werde nach den Parlamentswahlen den Entwurf eines Gesetzes vorstellen, das das Mehrheitswahlrecht auf allen Selbstverwaltungsebenen einführt.

\section{Frauen in den Exekutivorganen der territorialen Selbstverwaltung}

Die Einmandatswahlkreise gibt es in Polen bereits seit 2002, aufgrund des Gesetzes über die Direktwahl des Gemeindevorstehers (Gemeinden bis 20.000 Ew.), Bürgermeisters (Gemeinden bis 100.000 Ew.) und Stadtpräsidenten (Gemeinden ab 100.000 Ew.). Schaut man sich die Ergebnisse für Frauen bei diesen Wahlen an, lässt sich ihre minimale Repräsentanz in diesen lokalen Organen konstatieren. 2002 waren unter den gewählten Gemeindevorstehern, Bürgermeistern und Stadtpräsidenten nur 7 \% Frauen. Dieser Anteil stieg von Wahl zu Wahl, allerdings nur in minimalem Tempo - jeweils um 1 Prozentpunkt.

Eine direkte Ursache der eingeschränkten Repräsentanz von Frauen in den Exekutivorganen der territorialen Selbstverwaltung ist die geringe Anzahl von Kandidatinnen für diese Ämter. 2006 bemühten sich nur $12 \%$ Frauen um den Posten eines Gemeindevorstehers, Bürgermeisters oder Stadtpräsidenten, 2010 war dieser Anteil geringfügig auf $14 \%$ gestiegen.

Eine weitere Erklärung für diese schwache Partizipation von Frauen an der Exekutive auf allen Selbstverwaltungsebenen ist möglicherweise die begrenzte Rotation im Amt eines Gemeindevorstehers, Bürgermeisters oder Stadtpräsidenten. Bis zu 70 \% der 2010 Gewählten übten ihre Funktion auch schon vor den Wahlen aus. Wenn die Wähler in fast drei Vierteln der Kommunen die Personen wählen, die gerade das Amt ausüben, und unter diesen nur einen verschwindend geringen Anteil Frauen ausmachen (8\%), kann die Langsamkeit des Anstiegs der weiblichen Repräsentanz unter Gemeindevorstehern, Bürgermeistern und Stadtpräsidenten polnischer Städte nicht verwundern.

Der geringe Frauenanteil in den Exekutivorganen ist, was Untersuchungen auf allen höheren Selbstverwaltungsebenen bestätigen, auch mit der Einstellung 
zu erklären, Frauen seien nicht imstande zu leiten, d. h. schwierige Entscheidungen zu fällen oder einen Haushalt zu verwalten, die sowohl von Männern als auch von Frauen vertreten wird. Feldforschungen, die unter Leitung von Prof. Małgorzata Fuszara nach den Wahlen von 2002 und 2010 durchgeführt wurden, belegen, dass neben der Notwendigkeit, die häuslichen Pflichten zu erfüllen, die den Frauen eine breite Partizipation in der Politik unmöglich macht, eben solche Einstellungen über fehlende Führungsqualitäten eine wichtige Barriere auf dem Weg der Frauen in Ämter der Selbstverwaltungsorgane darstellen. Die in den Äußerungen der Befragten zum Vorschein kommende Reserve gegenüber der Ausfüllung politischer und leitender Funktionen durch Frauen bestätigen die Untersuchungen zur Präsenz von Frauen in den Exekutivorganen, nicht nur auf der Selbstverwaltungsebene.

In der Hälfte der polnischen Woiwodschaften gibt es keine Frau im Woiwodschaftsvorstand (zarząd województwa). In keinem Woiwodschaftsparlament stellen Frauen die Mehrheit. Von 16 Woiwodschaften wird nur in einer einzigen der Posten des Marschalls, also die Führungsposition, von einer Frau besetzt. Diese Situation ist nicht mit der Bindung der Wähler an die Amtsinhaber erklärbar, denn die Zusammensetzung der Leitungsgremien wird direkt von den Ratsmitgliedern des jeweiligen Regionalparlaments durch Wahl bestimmt. Noch nicht untersucht ist, inwieweit in diesem Zusammenhang die Tatsache von Bedeutung ist, dass $77 \%$ der Ratsmitglieder auf dieser Ebene Männer sind. Frauen sind jedoch zweifellos in der Hälfte der polnischen Regionen von der Beteiligung an für die Entwicklung der jeweiligen Woiwodschaft fundamentalen Entscheidungen ausgeschlossen. Fragen wie die Verteilung von EU-Mitteln, die Erarbeitung von regionalen Entwicklungsstrategien, die Haushaltsplanung oder Bewirtschaftung des Woiwodschaftseigentums - das sind nur einige der Aufgaben der Woiwodschaftsleitung. Der geringe Frauenanteil in diesen Entscheidungsgremien kann nicht mit der begrenzten Anzahl von Frauen im Regionalparlament selbst erklärt werden. Denn die Abgeordneten können die Mitglieder des Woiwodschaftsvorstands auch unter
Personen wählen, die nicht im Regionalparlament sitzen. Trotzdem befinden sich Frauen nur sehr selten in diesem Kreis.

\section{Zusammenfassung}

Die gleiche Möglichkeit aller Bürger und Bürgerinnen, am öffentlichen Leben der Gemeinschaft teilzuhaben, ist einer der Grundwerte der Demokratie. Ein Staat, in dem zumindest eine gesellschaftliche Gruppe bezüglich ihrer Partizipation am öffentlichen Leben diskriminiert wird, verfehlt das Gesamtinteresse der Gesellschaft, was sich nicht nur auf die diskriminierte Gruppe, sondern auch auf die Allgemeinheit negativ auswirkt. Die Steigerung des Frauenanteils in der Lokalpolitik dient daher nicht nur den Frauen selbst, die dadurch in der lokalen Gemeinschaft vollständige Gleichberechtigung erlangen, sondern vor allem den Organen der Kommunalverwaltung, die sich gleichmäßig entwickeln können, dank der unterschiedlichen Perspektiven, die bei der Entscheidungsfindung berücksichtigt werden. Jahrelange Untersuchungen der Teilnahme von Frauen an Wahlen zu den Selbstverwaltungsorganen zeigen, dass ihre Repräsentanz zunimmt, jedoch die 30\%-Marke, die so genannte kritische Masse nicht erreicht, die Frauen eine tatsächliche Einflussnahme auf die Angelegenheiten ihrer eigenen Region, ihres Kreises oder ihrer Stadt ermöglichen würde. Die besonders schwierige Situation von Frauen in den Exekutivorganen schränkt sie in ihrer vollständigen Teilhabe an der Kommunalpolitik ein. Quotenregelungen können den sich in den letzten Jahren verlangsamenden Anstieg des Anteils an weiblichen Mitgliedern in den Entscheidungsgremien wieder beschleunigen. $\mathrm{Zu}$ beobachten sind jedoch vor allem das Verhältnis der Parteien zur Platzierung von Frauen auf den vorderen Plätzen der Wahllisten und auch die Einstellungen der Frauen selbst zu einer Kandidatur bei lokalen Wahlen. Diese beiden Fragen erscheinen im Zusammenhang mit der Steigerung der politischen Partizipation von Frauen (nicht nur auf allen Ebenen der Selbstverwaltung) als die wichtigsten.

Übersetzung aus dem Polnischen: Ulrich Heiße

\section{Über die Autorinnen}

Małgorzata Druciarek ist Soziologin und forscht am Institut für öffentliche Angelegenheiten (Instytut Spraw Publicznych), Warschau.

Aleksandra Niżyńska ist Soziologin und forscht am Institut für öffentliche Angelegenheiten (Instytut Spraw Publicznych), Warschau; Doktorandin am Institut für angewandte Sozialwissenschaften der Universität Warschau. 


\section{Politische Partizipation von Frauen in Polen}

Grafik 1: Anteil der Kandidatinnen und Kandidaten auf den Wahllisten der einzelnen Parteien $2010(\%)$

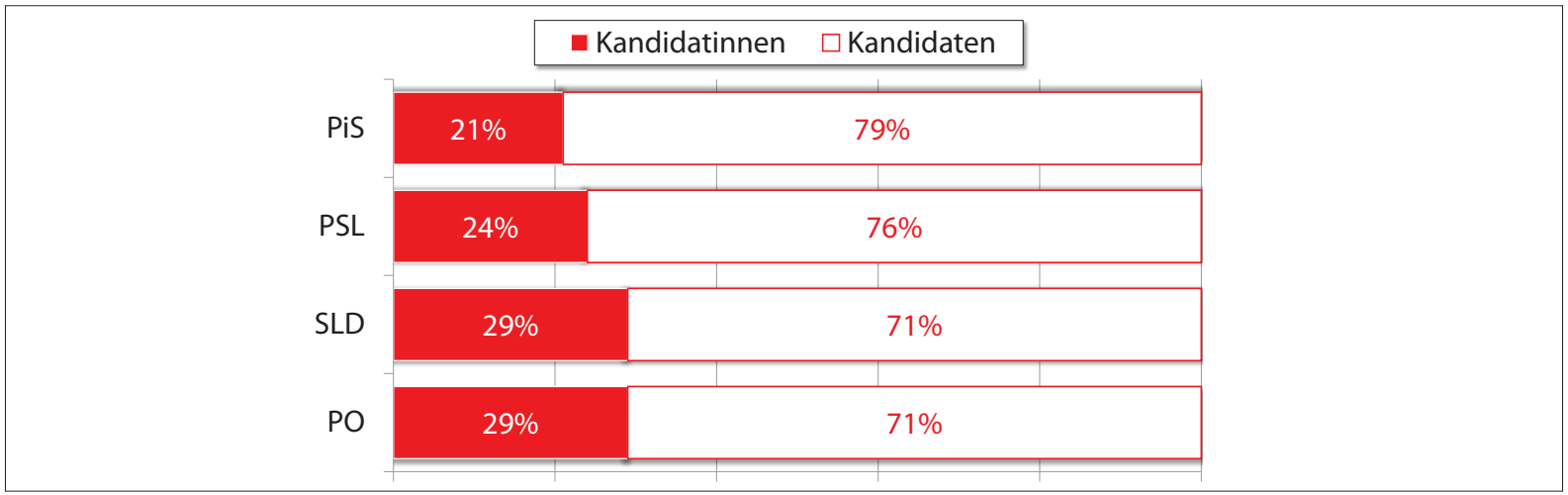

Quelle: Staatliche Wablkommission

Grafik 2: Frauen als Kandidatinnen und Ratsmitglieder in den Woiwodschaftsparlamenten $2010(\%)$

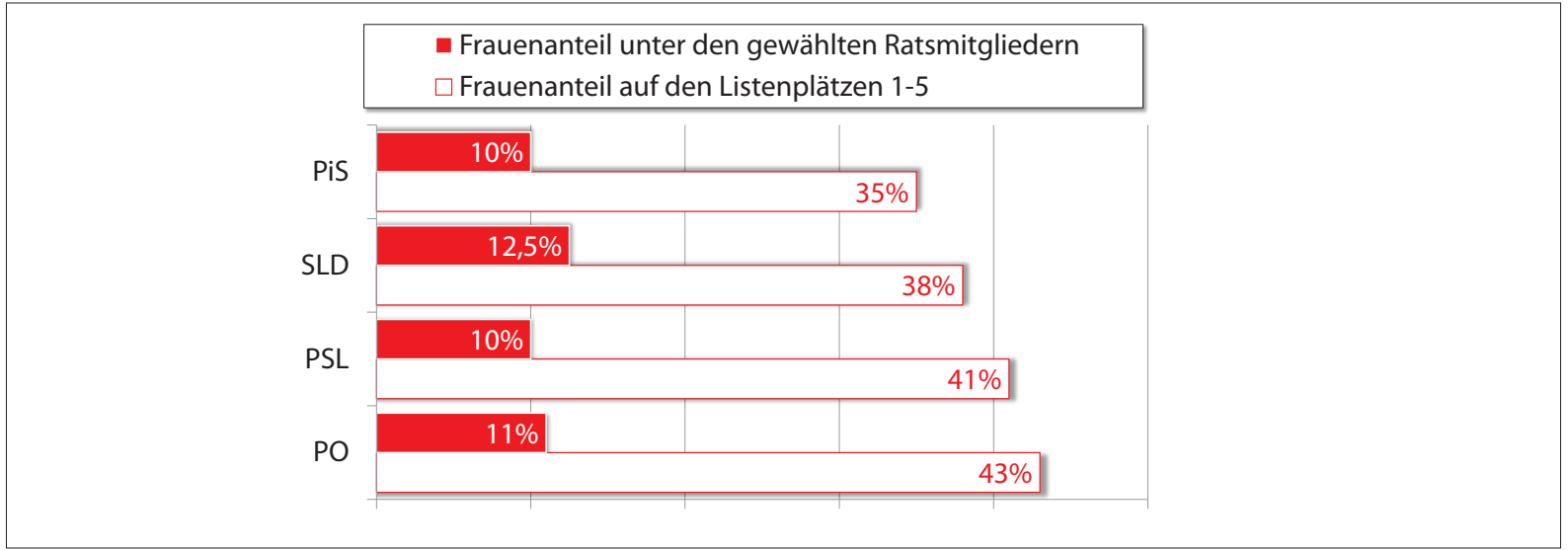

Quelle: Staatliche Wablkommission

Grafik 3: Anteil der Frauen und Männer auf den ersten Listenplätzen bei den Wahlen zu den Woiwodschaftsparlamenten 2010 (\%)

\begin{tabular}{|c|c|c|}
\hline & & ■ Frauen $\square$ Männer \\
\hline PiS & $11 \%$ & $89 \%$ \\
\hline SLD & $15 \%$ & $85 \%$ \\
\hline & & L \\
\hline PSL & $16 \%$ & $84 \%$ \\
\hline $\mathrm{PO}$ & $18 \%$ & $82 \%$ \\
\hline
\end{tabular}


Grafik 4: Anteil der Kandidatinnen auf Wahllisten von Parteien (\%)

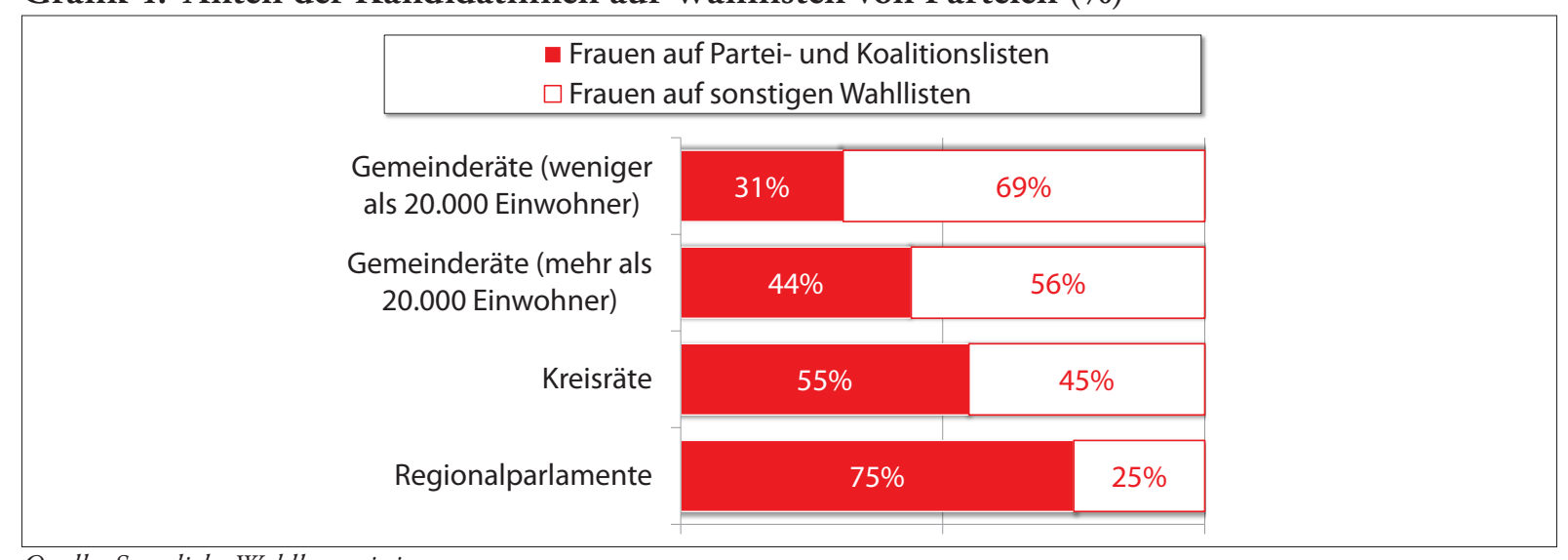

Quelle: Staatliche Wahlkommission

Grafik 5: Gewählte Ratsmitglieder auf den einzelnen Selbstverwaltungsebenen nach Geschlecht (\%)

\begin{tabular}{|c|c|c|}
\hline \multirow[b]{2}{*}{$\begin{array}{l}\text { Gemeinderäte (weniger } \\
\text { als } 20.000 \text { Einwohner) }\end{array}$} & \multicolumn{2}{|c|}{$\square$ Frauen } \\
\hline & $74,0 \%$ & $26,0 \%$ \\
\hline $\begin{array}{l}\text { Gemeinderäte (mehr als } \\
20.000 \text { Einwohner) }\end{array}$ & $78,4 \%$ & $21,6 \%$ \\
\hline $\begin{array}{c}\text { Stadträte der kreisfreien } \\
\text { Städte }\end{array}$ & $76,0 \%$ & $21,6 \%$ \\
\hline Kreisräte & $82,0 \%$ & $18,0 \%$ \\
\hline Regionalparlamente & $77,4 \%$ & $22,6 \%$ \\
\hline
\end{tabular}

Quelle: Staatliche Wahlkommission

Grafik 6: Anteil der Kandidatinnen zu den Woiwodschaftsparlamenten 2010 (\%)

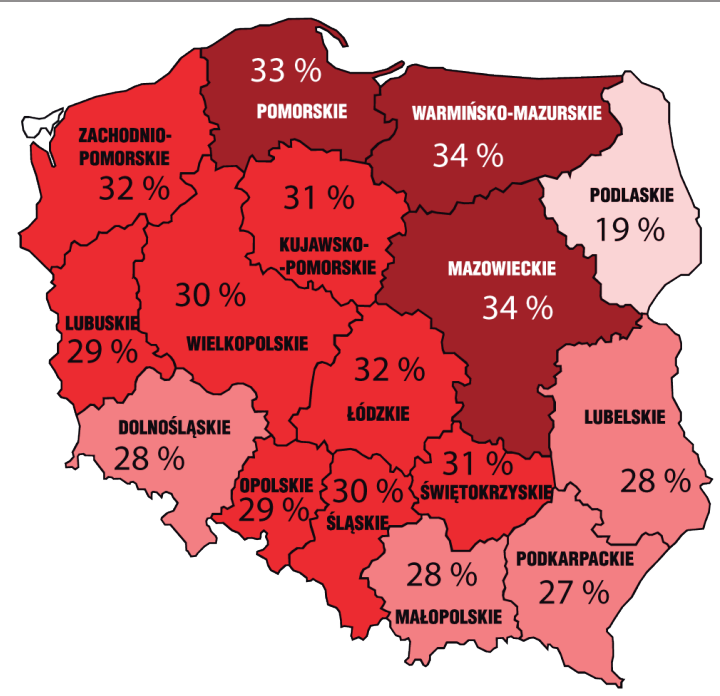

Quelle der Daten: Staatliche Wahlkommission

Karte: $C B O S, B S / 13 / 99$, Warszawa 01/1999. www.cbos.pl 
Grafik 7: Anteil der gewählten Frauen in den Woiwodschaftsparlamenten 2010

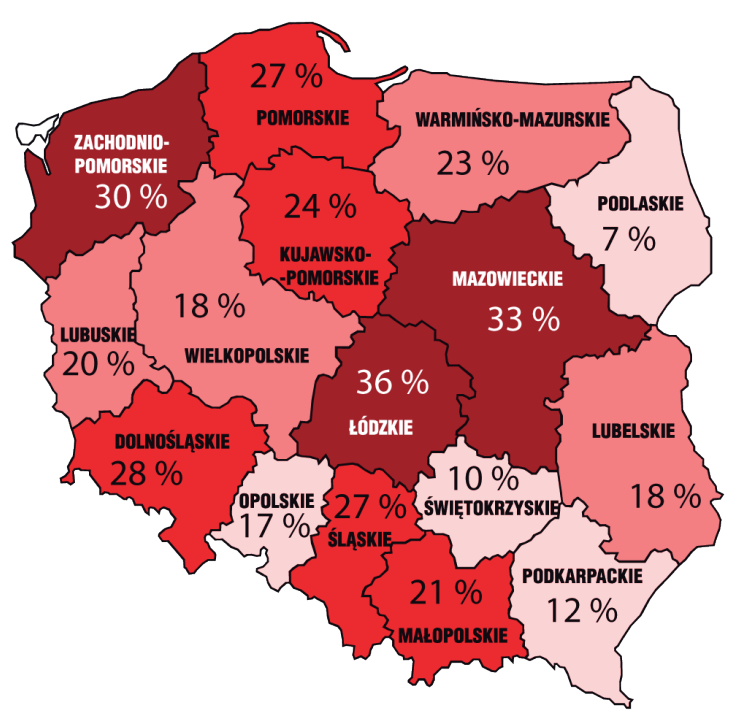

Quelle der Daten: Staatliche Wablkommission

Karte: CBOS, BS/13/99, Warszawa 01/1999. www.cbos.pl

Grafik 8: Anzahl der Frauen in den Woiwodschaftsvorständen 2010

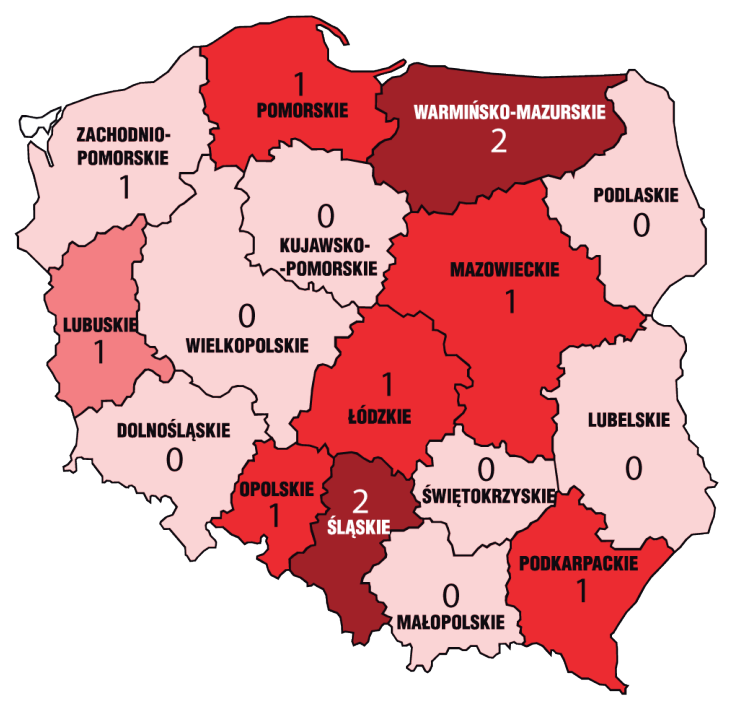

Quelle der Daten: Institut für Öffentliche Angelegenheiten [Instytut Spraw Publicznych] Karte: CBOS, BS/13/99, Warszawa 01/1999. www.cbos.pl 
Grafik 9: Anteil der Frauen in den regionalen Führungspositionen in Europa

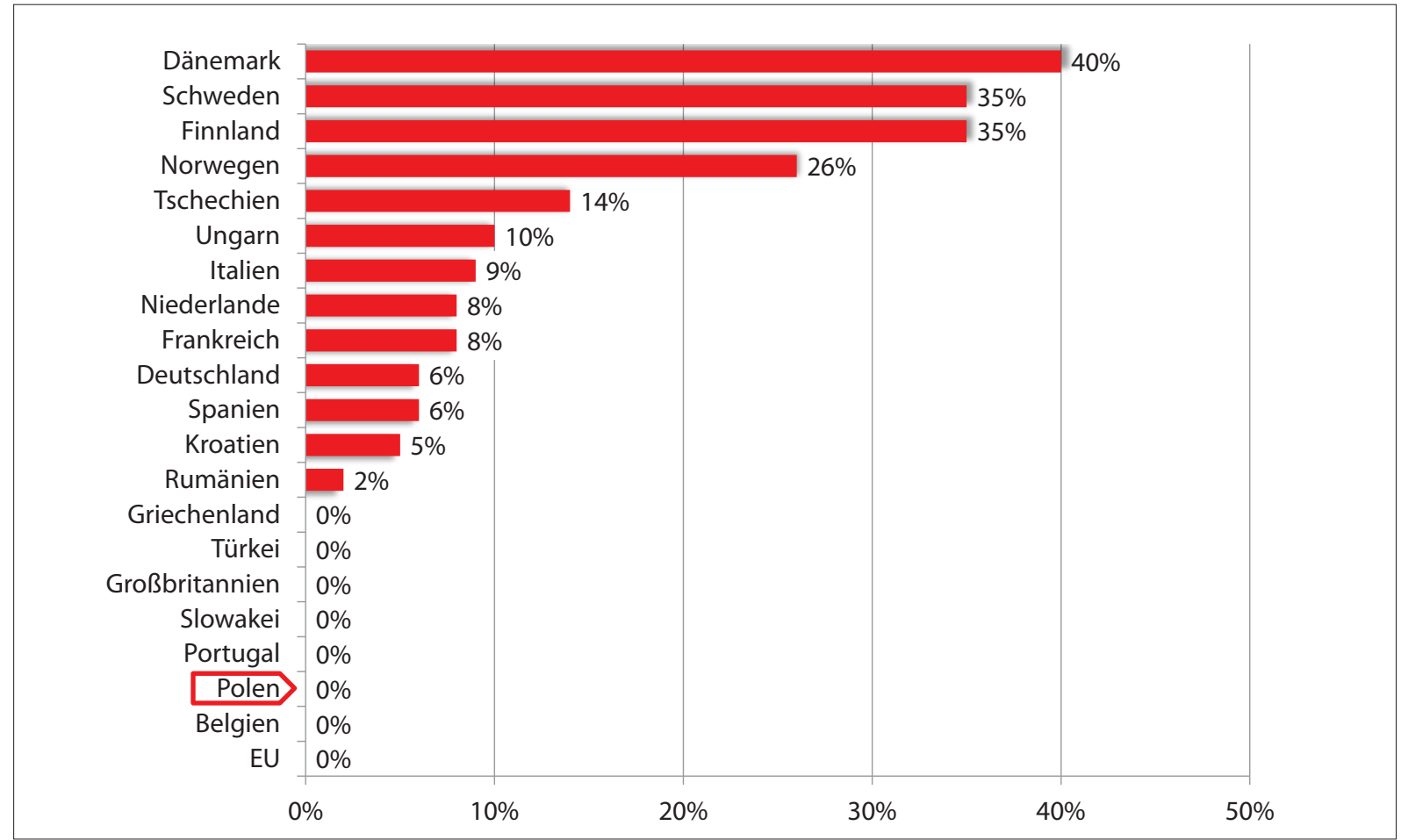

Quelle. Europäische Kommission 2010

\section{Grafik 10: Anteil der Frauen als Ratsmitglieder in den regionalen Parlamenten in Europa}

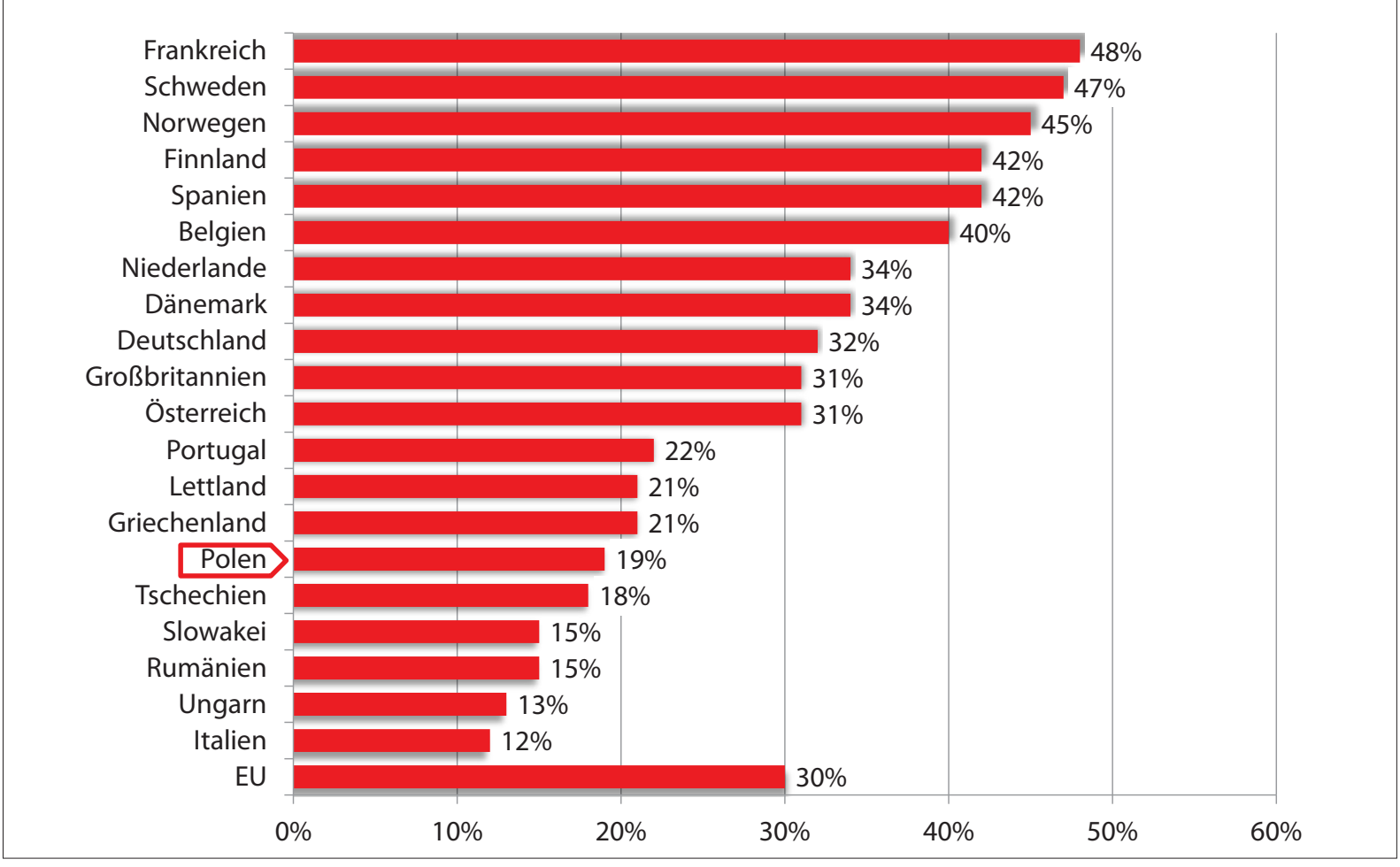

Quelle. Europäische Kommission 2010 
Grafik 11: Anteil der Frauen im Amt des Gemeindevorstehers/Bürgermeisters/Stadtpräsidenten in Europa

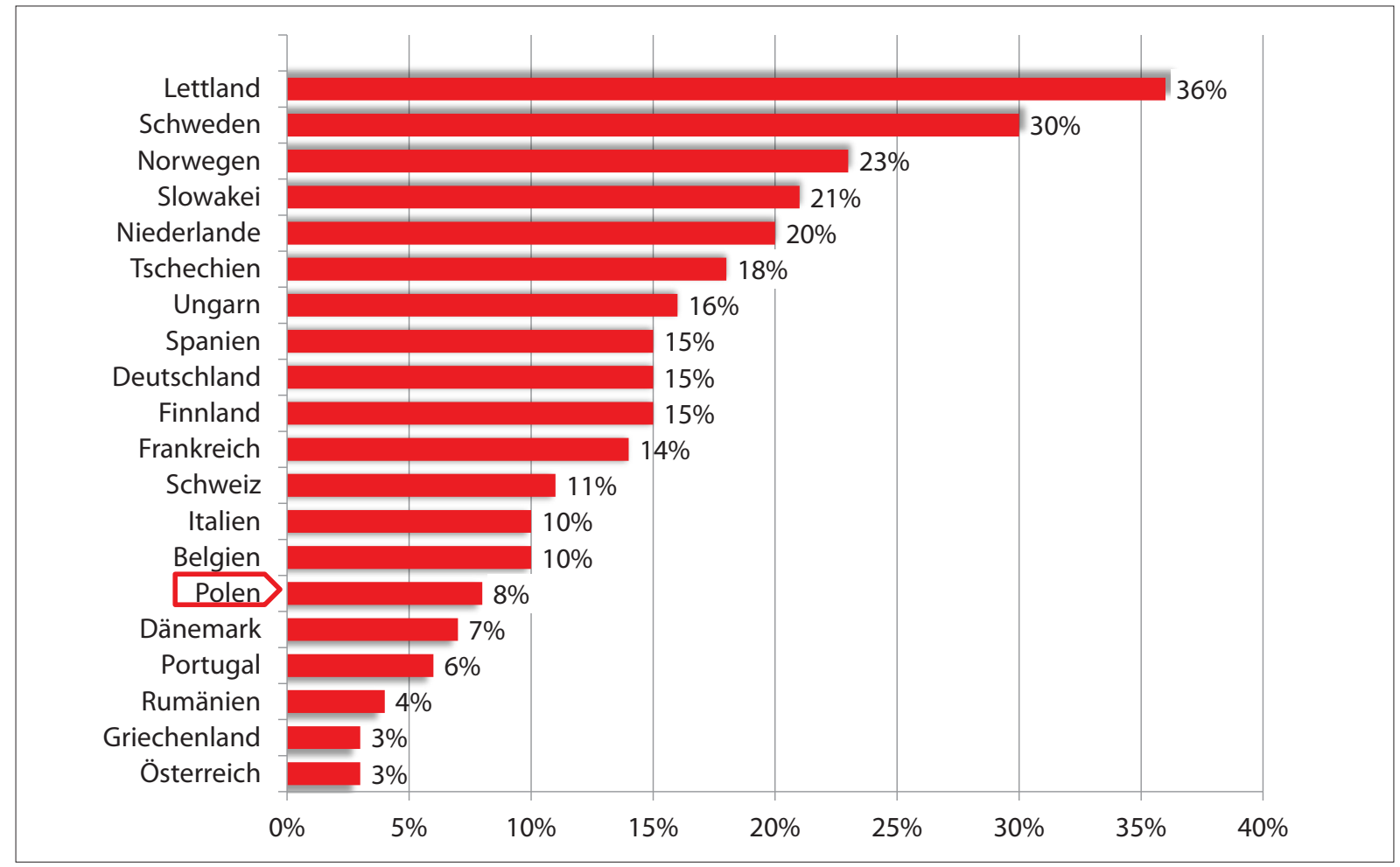

Quelle. Rat der Gemeinden und Regionen Europas 2008 


\section{Vom 20. September bis zum 03. Oktober 2011}

\begin{tabular}{|c|c|}
\hline 20.09 .2011 & $\begin{array}{l}\text { Die Regierung beschließt die Verkleinerung des polnischen Kontingents in Afghanistan, d. h. eine Reduzierung } \\
\text { um } 100 \text { Personen in Afghanistan und um } 200 \text { Personen in der Reserve in Polen. Damit würden } 2.500 \text { Perso- } \\
\text { nen im Einsatz stehen und } 200 \text { Personen der Reserve angehören. }\end{array}$ \\
\hline 21.09 .2011 & $\begin{array}{l}\text { Der Unterstaatssekretär im Handelsdepartement der USA, Francisco Sanchez, betont während seines Besuchs } \\
\text { in Polen das Interesse der US-amerikanischen Regierung sowie der Privatwirtschaft, mit Polen im Bereich der } \\
\text { Atomenergie zusammenzuarbeiten. }\end{array}$ \\
\hline 21.09 .2011 & $\begin{array}{l}\text { Bundespräsident Christian Wulff und Staatspräsident Bronisław Komorowski eröffnen in Berlin die Ausstellung } \\
\text { »Tür an Tür. Polen - Deutschland. } 1000 \text { Jahre Kunst und Geschichte» im Gropiusbau. }\end{array}$ \\
\hline 23.09 .2011 & $\begin{array}{l}\text { Außenminister Radosław Sikorski teilt mit, dass er in Absprache mit der hohen Vertreterin der EU für Außen- } \\
\text { und Sicherheitspolitik den belarussischen Außenminister zum Gipfel der Östlichen Partnerschaft in War- } \\
\text { schau in der kommenden Woche eingeladen hat. Der Gipfel wird von der polnischen Ratspräsidentschaft aus- } \\
\text { gerichtet. Sikorski unterstreicht, dass Polen dafür eintritt, dass sich Belarus den freien europäischen Nationen } \\
\text { anschließt. Eine Bedingung für den Dialog mit Belarus sei, dass die politischen Gefangenen freigelassen und } \\
\text { rehabilitiert werden. }\end{array}$ \\
\hline 24.09 .2011 & $\begin{array}{l}\text { Auf ihren Wahlkampfveranstaltungen für die Parlamentswahlen am 9. Oktober sprechen sich Ministerpräsi- } \\
\text { dent Donald Tusk und der Vorsitzende von Recht und Gerechtigkeit (Prawo i Sprawiedliwość - PiS), Jarosław } \\
\text { Kaczyński, gleichermaßen gegen Steuersenkungen aus. }\end{array}$ \\
\hline 25.09 .2011 & $\begin{array}{l}\text { Staatspräsident Bronisław Komorowski unterschreibt das Gesetz über die Zugänglichkeit öffentlicher Informa- } \\
\text { tionen. Es sieht vor, dass die Freigabe von Informationen für die Öffentlichkeit dann eingeschränkt wird, wenn } \\
\text { es um den Schutz eines wichtigen wirtschaftlichen Interesses des Staates geht, beispielsweise bei Verhandlun- } \\
\text { gen im Rahmen von EU- oder anderen internationalen Verträgen. Nichtregierungsorganisationen und Opposi- } \\
\text { tion hatten an Komorowski appelliert, ein Veto einzulegen. Zur Überprüfung der Verfassungsmäßigkeit leitet } \\
\text { Komorowski das Gesetz am 28.09.2011 an das Verfassungsgericht weiter. }\end{array}$ \\
\hline 26.09 & $\begin{array}{l}\text { Der Vorsitzende der Unabhängigen Selbstverwalteten Gewerkschaft Solidarność (Niezależny Samorządny Zwią- } \\
\text { zek Zawodowy - NSZZ Solidarność), Piotr Duda, appelliert in einem Brief an Ministerpräsident Donald Tusk, } \\
\text { eine gemeinsame Kommission der Regierung und der Gewerkschaft einzurichten, die sich mit der Umsetzung des } \\
\text { sogenannten EU-Klimapakets (2008) befassen soll. Duda warnt, dass die Umsetzung der Richtlinien des EU-Kli- } \\
\text { mapakets zu starken Preissteigerungen für Energie und Wärme ab } 2013 \text { führen würde. Dies werde eine erhebliche } \\
\text { Belastung für die Verbraucher und die Betriebe werden und den Anstieg der Arbeitslosigkeit nach sich ziehen. }\end{array}$ \\
\hline 27.0 & $\begin{array}{l}\text { Die Regierung verabschiedet den Haushalt für 2012. Ausgegangen } \\
\text { Mrd. Zloty und Einnahmen in Höhe von zirka } 293 \text { Mrd. Zloty. }\end{array}$ \\
\hline 28.09 & onelle zum 35. Jahrestag der Gründung des Komi- \\
\hline 29.09 .2011 & $\begin{array}{l}\text { In Warschau beginnt der EU-Gipfel zur Östlichen Partnerschaft. Ministerpräsident Tusk stellt Belarus die Unter- } \\
\text { stützung der Reformprozesse zur Beschleunigung der politischen und wirtschaftlichen Integration mit der EU, } \\
\text { ein Assoziierungsabkommen und Verhandlungen zu Visa-Erleichterungen sowie die Erweiterung des Jugendaus- } \\
\text { tausches in Aussicht. Im Gegenzug müsste das Land seine rechtsstaatlichen und marktwirtschaftlichen Refor- } \\
\text { men beschleunigen. Bundeskanzlerin Merkel trifft am Rande des Gipfels belarussische Oppositionelle. Dabei } \\
\text { fordert sie die Freilassung aller politischen Gefangenen. }\end{array}$ \\
\hline \begin{tabular}{|l|}
30.09 .2011 \\
\end{tabular} & $\begin{array}{l}\text { Auf dem zweiten und letzten Tag des EU-Gipfels zur Östlichen Partnerschaft in Warschau steht Belarus trotz } \\
\text { seines Boykotts der Tagung im Zentrum der Beratungen. Die EU bietet Minsk } 9 \text { Mrd. Euro und die Aufhe- } \\
\text { bung der Visapflicht an, wenn die Regierung politische Gefangene frei lässt, mit der Opposition spricht und } \\
\text { freie Wahlen erlaubt. Die Vertreter der anwesenden osteuropäischen Partnerschaftsländer (Ukraine u. a.) hal- } \\
\text { ten sich mit direkter Kritik des Lukaschenka-Regimes zurück und sind nicht bereit, das Abschlussdokument } \\
\text { zu unterzeichnen. Darüber hinaus will die EU bis Dezember bilaterale Verträge abschließen, die Handels- und } \\
\text { Visa-Angelegenheiten regeln. }\end{array}$ \\
\hline 02.10.2011 & $\begin{array}{l}\text { Der renommierte Nike-Literaturpreis geht an Marian Pilot, einen Vertreter der Bauernkultur in der Literatur. } \\
\text { Sein Werk »Pióropusz« (Federbusch), das ausgezeichnet wurde, verweist auf einen Pelikan-Federhalter, der als } \\
\text { Geschenk des Großvaters den Protagonisten auf dem Weg in die Kindheit in einem Dorf namens Siedlikowo } \\
\text { begleitet. }\end{array}$ \\
\hline 03.10 .2011 & $\begin{array}{l}\text { Die polnische Nationalbank verkauft Euro in großem Umfang, um den Zloty zu stabilisieren. Von den Kursen } \\
\text { am Freitag (30.09.) und Montag (03.10.) hängen die Preise für Benzin, die Höhe der EU-Direktbeihilfen für } \\
\text { die Bauern sowie der Umfang zahlreicher steuerlicher Vergünstigungen für Unternehmen im nächsten Jahr ab. }\end{array}$ \\
\hline
\end{tabular}


Die Polen-Analysen erscheinen zweimal monatlich als E-Mail-Dienst. Sie werden gemeinsam vom Deutschen PolenInstitut Darmstadt, der Bremer Forschungsstelle Osteuropa und der Deutschen Gesellschaft für Osteuropakunde herausgegeben.

Ein Archiv der Polen-Analysen finden Sie im Internet unter www.laender-analysen.de/polen

Kostenloses Abonnement unter http://www.deutsches-polen-institut.de/Newsletter/subscribe.php

Diese Analysen finden Sie online als Lizenzausgabe auf

bpb.de



\section{Deutsches Polen-Institut Darmstadt}

Das Deutsche Polen-Institut Darmstadt (DPI) ist ein Forschungs-, Informations-, und Veranstaltungszentrum für polnische Kultur, Geschichte, Politik, Gesellschaft und die deutsch-polnischen Beziehungen, die sich im Kontext der europäischen Integration entwickeln. Das seit März 1980 aktive und bis 1997 von Gründungsdirektor Karl Dedecius geleitete Institut ist eine Gemeinschaftsgründung der Stadt Darmstadt, der Länder Hessen und Rheinland-Pfalz sowie des Bundes. 1987 wurden die Kultusminister der Länder und 2011 das Auswärtige Amt weitere institutionelle Träger. Einen wesentlichen Beitrag zur Verwirklichung der Institutsziele leisten private Stiftungen. Das DPI hat satzungsgemäß die Aufgabe, durch seine Arbeit zur Vertiefung der gegenseitigen Kenntnisse des kulturellen, geistigen und gesellschaftlichen Lebens von Polen und Deutschen beizutragen.

Ziel der Vermittlertätigkeit des DPI ist es, „die zu interessieren, auf die es politisch, wirtschaftlich, gesellschaftlich und kulturell im deutsch-polnischen Verhältnis ankommt« (Leitlinien 1997). Es geht um die Entscheider und Multiplikatoren in Politik, Kultur, Bildung, Verwaltung, Medien und Wirtschaft und, wesentlich stärker ausgeprägt als bisher, um das Hineinwirken in Wissenschaft, Forschung und Bildung.

Derzeit bemüht sich das DPI in Kooperation mit den verstreuten Orten wissenschaftlicher Polen-Kompetenz an deutschen Hochschulen und Forschungsinstituten verstärkt darum, ausgehend von einer Bestandsaufnahme deutscher Polen-Forschung Ort wissenschaftlicher Forschung und verbindendes, vernetzendes und kooperierendes Zentrum zu werden. Ausgangspunkt der Neuausrichtung ist die kaum mehr kontrollierbare Dynamik des Rückbaus der Ressourcen der wissenschaftlichen Polen-Kompetenz in den unterschiedlichen Disziplinen. Mit der knapp 60.000 Bände zählenden multidisziplinären Fachbibliothek für Polen, die eine einzigartige Sammlung polnischer Literatur in der Originalsprache und in deutscher Übersetzung umfasst, ist das DPI bereits ein geschätzter Ort der Recherche und des wissenschaftlichen Arbeitens. (www.deutsches-polen-institut.de)

\section{Forschungsstelle Osteuropa an der Universität Bremen}

1982 gegründet, widmet sich die Forschungsstelle Osteuropa an der Universität Bremen kulturellen und gesellschaftlichen Entwicklungen der Länder Ost- und Ostmitteleuropas in Zeitgeschichte und Gegenwart. Die Forschungsstelle besitzt in ihrem Archiv eine einzigartige Sammlung alternativer Kulturgüter und unabhängiger Texte aus den ehemaligen sozialistischen Ländern. Darunter befindet sich auch eine umfangreiche Sammlung des "Zweiten Umlaufs«, die das Schrifttum und Dokumente unabhängiger Initiativen und gesellschaftlicher Gruppen in Polen aus der Zeit von 1976 bis zum Umbruch umfasst. Neben ausführlicher individueller Forschung zu Dissens und Gesellschaft im Sozialismus, leitet die Forschungsstelle seit Januar 2007 ein gemeinsames Projekt mit einem Verbund von internationalen Forschungsinstituten zum Thema "Das andere Osteuropa - die 1960er bis 1980er Jahre, Dissens in Politik und Gesellschaft, Alternativen in der Kultur. Beiträge zu einer vergleichenden Zeitgeschichte«, welches von der VolkswagenStiftung finanziert wird.

Im Bereich der post-sozialistischen Gesellschaften sind in den letzten Jahren umfangreiche Forschungsprojekte durchgeführt worden, deren Schwerpunkte auf politischen Entscheidungsprozessen, Wirtschaftskultur und der EU-Osterweiterung lagen. Eine der Hauptaufgaben der Forschungsstelle ist die Information der interessierten Öffentlichkeit. Dazu gehören unter anderem regelmäßige E-Mail-Informationsdienste mit fast 15.000 Abonnenten in Politik, Wirtschaft und den Medien.

Mit ihrer in Deutschland einzigartigen Sammlung von Publikationen zu Osteuropa ist die Forschungsstelle eine Anlaufstelle sowohl für Wissenschaftler als auch für die interessierte Öffentlichkeit. In der Bibliothek sind derzeit neben anderen breit angelegten Beständen allein aus Polen ca. 300 laufende Periodika zugänglich. Die Bestände werden in Datenbanken systematisch erfasst. (www. forschungsstelle.uni-bremen.de)

Die Meinungen, die in den Polen-Analysen geäußert werden, geben ausschließlich die Auffassung der Autoren wieder. Abdruck und sonstige publizistische Nutzung sind nach Rücksprache mit der Redaktion gestattet. Redaktion: Prof. Dr. Dieter Bingen (Darmstadt), Silke Plate, M.A. (Bremen) Technische Gestaltung: Matthias Neumann

Polen-Analysen-Layout: Cengiz Kibaroglu, Matthias Neumann

Die Polen-Analysen werden im Rahmen der Datenbank World Affairs Online (WAO) ausgewertet und sind im Portal IREON www.ireon-portal.de recherchierbar.

ISSN 1863-9712 @ 2011 by Deutsches Polen-Institut Darmstadt und Forschungsstelle Osteuropa, Bremen

Kontakt: Dr. Andrzej Kaluza, Presse- und Öffentlichkeitsarbeit, Deutsches Polen-Institut, Mathildenhöhweg 2,

D-64287 Darmstadt, Tel.: 06151/4985-13, Fax: 06151/4985-10, E-Mail: polen-analysen@dpi-da.de, Internet: www.laender-analysen.de/polen 